\title{
VTLN-BASED VOICE CONVERSION
}

\author{
David Sündermann and Hermann Ney \\ RWTH Aachen - University of Technology \\ Computer Science Department \\ Ahornstr. 55, 52056 Aachen, Germany \\ $\{$ suendermann, ney\}acs.rwth-aachen. de
}

\begin{abstract}
In speech recognition, vocal tract length normalization (VTLN) is a well-studied technique for speaker normalization. As voice conversion aims at the transformation of a source speaker's voice into that of a target speaker, we want to investigate whether VTLN is an appropriate method to adapt the voice characteristics. After applying several conventional VTLN warping functions, we extend the piecewise linear function to several segments, allowing a more detailed warping of the source spectrum. Experiments on voice conversion are performed on three corpora of two languages and both speaker genders.
\end{abstract}

\section{INTRODUCTION}

Vocal tract length normalization [1] tries to compensate for the effect of speaker dependent vocal tract lengths by warping the frequency axis of the amplitude spectrum. In speech recognition, VTLN aims at the normalization of a speaker's voice in order to remove individual speaker characteristics.

A similar task is voice conversion. It describes the modification of a source speaker's voice such that it is perceived to be spoken by a target speaker [2]. In this paper, we show how VTLN can be applied to this task.

In Section 2, we delineate a method to find corresponding speech segments respectively artificial phonetic classes in the training material of the source and the target speaker. These corresponding classes are used to estimate the parameters of class-dependent VTLN warping functions. Subsequently, in Section 3, we apply this training procedure to conventional warping functions depending on only one parameter.

Often, these conventional functions do not sufficiently model the speakers' characteristics. Therefore, we introduce a piece-wise linear warping function consisting of several linear segments. The greater the parameter number is, the more carefully we must deal with their practical estimation. All these considerations are discussed in Section 4.

Since the parameter estimation for classes with only few observations can be very inaccurate and, besides, we do not want the parameters to change abruptly from one class to another, in Section 5, we introduce two parameter smoothing methods. Finally, in Section 6, we present experimental results on three German and English corpora.

\section{AUTOMATIC SEGMENTATION AND MAPPING}

Most of the training procedures of state-of-the-art voice conversion techniques require training data containing the same utterances of both source and target speaker [3]. Besides, these utterances should feature a high degree of natural time alignment and similar pitch contour [4].

However, in several voice conversion applications (e. g. spontaneous speaker adaptation or speech-to-speech translation) we do not possess corresponding time frames of source and target speaker. In [5], we address this problem as follows.

At first, we subdivide speech material of speaker $S$ and $T$ into $K_{S}$ respectively $K_{T}$ artificial phonetic classes. This is done by clustering the frequency spectra of period-synchronous frames obtained by a pitch tracker. For unvoiced signal parts, pseudo periods are used. Now, for each source class $k_{S}$ we determine the most similar target class $\hat{k}_{T}\left(k_{S}\right)$. This class mapping is basis for an arbitrary statistical voice conversion parameter training.

\subsection{Statistical Voice Conversion Parameter Training}

Let $X_{1}^{I}=X_{1}, \ldots, X_{I}$ be the spectra belonging to source class $k_{S}$ and $Y_{1}^{J}$ those of the mapped class $\hat{k}_{T}\left(k_{S}\right)$, we generally estimate the parameter vector $\vartheta$ by minimizing the sum of the euclidean distances between all target class spectra and transformed source class spectra. Here, we utilize the spectral conversion function $F_{\vartheta^{\prime}}$ depending on the parameter vector $\vartheta^{\prime}$.

$$
\vartheta=\arg \min _{\vartheta^{\prime}} \sum_{i=1}^{I} \sum_{j=1}^{J} \int_{\omega=0}^{\pi}\left|Y_{j}(\omega)-F_{\vartheta^{\prime}}\left(X_{i}, \omega\right)\right|^{2} d \omega
$$

In conjunction with a suitable smoothing technique, we often can neglect the variety of the classes' observation spec- 
tra by introducing a mean approximation without an essential effect on the voice conversion parameters.

$$
\vartheta=\arg \min _{\vartheta^{\prime}} \int_{\omega=0}^{\pi}\left|\bar{Y}(\omega)-F_{\vartheta^{\prime}}(\bar{X}, \omega)\right|^{2} d \omega
$$

Here, $\bar{X}$ and $\bar{Y}$ are the source and target classes' average spectra.

\section{WARPING FUNCTIONS WITH ONE PARAMATER}

In speech recognition, several VTLN warping functions have been proposed whose parameters usually are limited to one variable, the warping factor $\alpha$. Established warping functions are

- the symmetric piece-wise linear function with two segments [6]

$$
\begin{gathered}
\tilde{\omega}_{\alpha}(\omega)=\left\{\begin{array}{cc}
\alpha \omega & : \omega \leq \omega_{0} \\
\alpha \omega_{0}+\frac{\pi-\alpha \omega_{0}}{\pi-\omega_{0}}\left(\omega-\omega_{0}\right) & : \quad \omega \geq \omega_{0}
\end{array}\right. \\
\omega_{0}=\left\{\begin{array}{cc}
\frac{7}{8} \pi & : \quad \alpha \leq 1 \\
\frac{7}{8 \alpha} \pi & : \quad \alpha \geq 1
\end{array}\right.
\end{gathered}
$$

- the power function [7]

$$
\tilde{\omega}_{\alpha}(\omega)=\left(\frac{\omega}{\pi}\right)^{\alpha}
$$

- the quadratic function [8]

$$
\tilde{\omega}_{\alpha}(\omega)=\omega+\alpha\left(\frac{\omega}{\pi}-\left(\frac{\omega}{\pi}\right)^{2}\right)
$$

- the bilinear function [9]

$$
\tilde{z}_{\alpha}(z)=\frac{z-\alpha}{1-\alpha z} \quad \text { with } \quad z=e^{i \omega}
$$

In order to estimate the class dependent warping factor $\alpha$, we use Eqs. 1 or 2, where

$$
F_{\alpha}(X, \omega)=X\left(\tilde{\omega}_{\alpha}(\omega)\right) .
$$

\section{WARPING FUNCTIONS WITH SEVERAL PARAMETERS}

\subsection{Piece-Wise Linear Warping with Several Segments}

One of the adversarial properties of the conventional warping functions with one parameter is that the whole frequency axis is always warped in the same direction, either to lower or to higher frequencies. Consequently, these functions are not able to model spectral conversions where certain parts of the axis move to higher frequencies, and other parts to lower frequencies, or vice versa. Such functions would require at least one inflection point and would cross the $\tilde{\omega}=\omega$ diagonal.

Applying the VTLN technique to voice conversion, we want to use more exact models than in speech recognition, i. e. warping functions with several parameters, for a better description of the individual characteristics of the speakers' vocal tracts.

Assuming there is an ideal warping function for a given class pair $\left(k_{S}, \hat{k}_{T}\right)$, an obvious model is given by the interpolation of this function by several linear segments, as a consequence from the simple two-segment linear warping, vide Eq. 3.

$$
\begin{gathered}
\tilde{\omega}_{\tilde{\omega}_{1}^{S}}(\omega)=\left\{\begin{array}{ccc}
\tilde{\omega}_{0, \tilde{\omega}_{1}}(\omega) & \text { for } & 0 \leq \omega \leq \frac{1}{S+1} \cdot \pi \\
\vdots & & \vdots \\
\tilde{\omega}_{\tilde{\omega}_{s}, \tilde{\omega}_{s+1}}(\omega) & \text { for } & \frac{s}{S+1} \cdot \pi \leq \omega \leq \frac{s+1}{S+1} \cdot \pi \\
\vdots & & \vdots \\
\tilde{\omega}_{\tilde{\omega}_{S}, \pi}(\omega) & \text { for } & \frac{S}{S+1} \cdot \pi \leq \omega \leq \pi
\end{array}\right. \\
\tilde{\omega}_{\tilde{\omega}^{\prime}, \tilde{\omega}^{\prime \prime}}(\omega)=\tilde{\omega}^{\prime}+\left(\frac{S+1}{\pi} \cdot \omega-s\right) \cdot\left(\tilde{\omega}^{\prime \prime}-\tilde{\omega}^{\prime}\right) \\
0 \leq \tilde{\omega}_{1} \leq \cdots \leq \tilde{\omega}_{S} \leq \pi .
\end{gathered}
$$

This formula describes a piece-wise linear function $\tilde{\omega}(\omega)$ starting at $(0,0)$, ending at $(\pi, \pi)$, and connecting $S$ points whose $\omega$ values are equidistantly distributed. The corresponding $\tilde{\omega}_{s}$ are the parameters of the warping function. The resulting function is monotonous according to Eq. 7, as we do not want parts of the frequency axis to be exchanged.

\subsection{Practical Parameter Estimation}

In general, augmenting the number of parameters confronts us with an increasing need of computation time. Particularly, this is the case if the minimization of Eqs. 1 or 2 is performed by calculating the distances for all possible parameter combinations concerning a certain resolution. This estimation method results in an exponential increase of computing time in dependence on the number of considered parameters.

Viewing the definition of the piece-wise linear warping function with several segments, cf. Eq. 6, we note that the integrals used in Eqs. 1 and 2 can be rewritten as (also cp. Eq. 5)

$$
\begin{aligned}
d_{\tilde{\omega}_{1}^{S}} & =\int_{\omega=0}^{\pi}\left|Y(\omega)-X\left(\tilde{\omega}_{\tilde{\omega}_{1}^{S}}(\omega)\right)\right|^{2} d \omega \\
& =\sum_{s=0}^{S} \int_{\omega=\frac{s}{S+1} \cdot \pi}^{\frac{s+1}{S+1} \cdot \pi}\left|Y(\omega)-X\left(\tilde{\omega}_{\tilde{\omega}_{s}^{s+1}}(\omega)\right)\right|^{2} d \omega .
\end{aligned}
$$


This enables us to use dynamic programming for searching the minimum distance and therewith the optimal parameter vector $\tilde{\omega}_{1}^{S}$.

\section{PARAMETER SMOOTHING}

\subsection{Iterative Integrating Smoothing}

Basis of the voice conversion technique delineated in this paper is the automatic class segmentation and mapping described in Section 2. In Figure 1, we show the time course of the word "Arizona" and the corresponding classes for $K_{S}=8$.

To avoid that the class-dependent voice conversion parameters jump at the class boundaries causing distinctly audible artifacts in the converted speech, we introduce an integrating parameter smoothing which iteratively adapts a parameter vector by adding a weighted mean of the chronologically neighbored vectors. Figure 2 shows the effect of this smoothing technique for 5, 50 and 5000 iterations using the symmetric piece-wise warping function described in Eq. 3. If the number of iterations approaches infinity, we obtain a constant function over the time representing the mean parameter vector.

\subsection{Deviation Penalty}

Viewing Figures 1 and 2, we note that for certain classes the obtained parameter values highly deviate from the mean. E. g. for $k_{S}=7$ we obtain an $\alpha$ less than 1 , whereas the particular voice conversion (female-male) should result in values greater than 1 . Considering the mean of $\bar{\alpha}=1.3$, the parameter values are to be controlled and, if necessary, corrected towards the mean.

This is performed by applying the minimization Eqs. 1 or 2 a second time, having added a penalty term to the enclosed integral. Both addends are normalized by their maximum and then weighted utilizing the real value $0 \leq \lambda \leq 1$ to adjust the penalty strength. Hence, $\lambda=1$ does not influence the class parameters at all, whereas $\lambda=0$ forces all parameters to be equal to their mean $\bar{\vartheta}$. An equilibrium between both terms is to be around $\lambda=0.5$.

In the following, we assume $X$ and $Y$ to have the unity energy $E_{0}$ in order to remove the dependence of the distances on the signal loudness.

$$
\begin{aligned}
& d_{\vartheta}= \lambda \frac{\int_{\omega=0}^{\pi}\left|Y(w)-X\left(\tilde{\omega}_{\vartheta}(\omega)\right)\right|^{2} d \omega}{\max _{X^{\prime}, Y^{\prime}} \int_{\omega=0}^{\pi}\left|Y^{\prime}(w)-X^{\prime}(\omega)\right|^{2} d \omega} \\
&+(1-\lambda) \frac{\int_{\omega=0}^{\pi}\left(\tilde{\omega}_{\bar{\vartheta}}(\omega)-\tilde{\omega}_{\vartheta}(\omega)\right)^{2} d \omega}{\max _{\bar{\vartheta}^{\prime}, \vartheta^{\prime}} \int_{\omega=0}^{\pi}\left(\tilde{\omega}_{\bar{\vartheta}^{\prime}}(\omega)-\tilde{\omega}_{\vartheta^{\prime}}(\omega)\right)^{2} d \omega}
\end{aligned}
$$

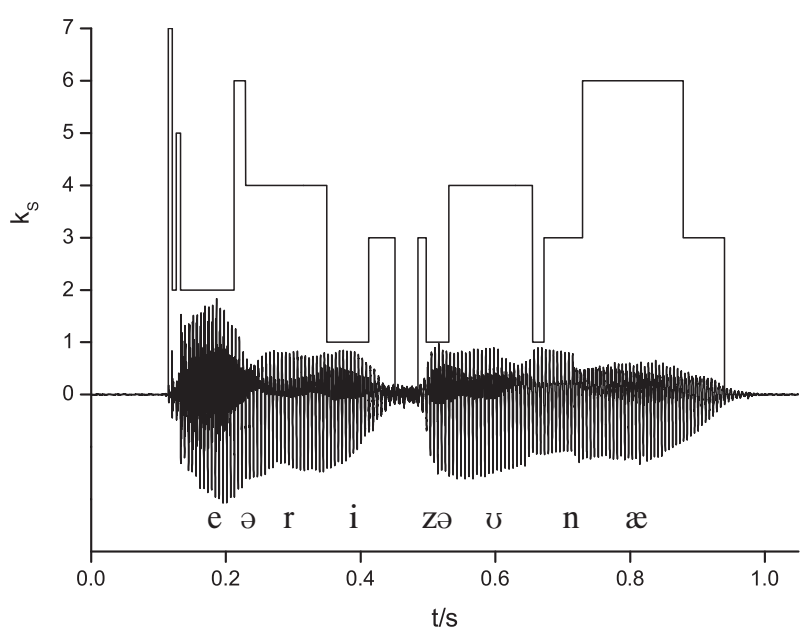

Fig. 1. Automatic Class Segmentation for the Word "Arizona".

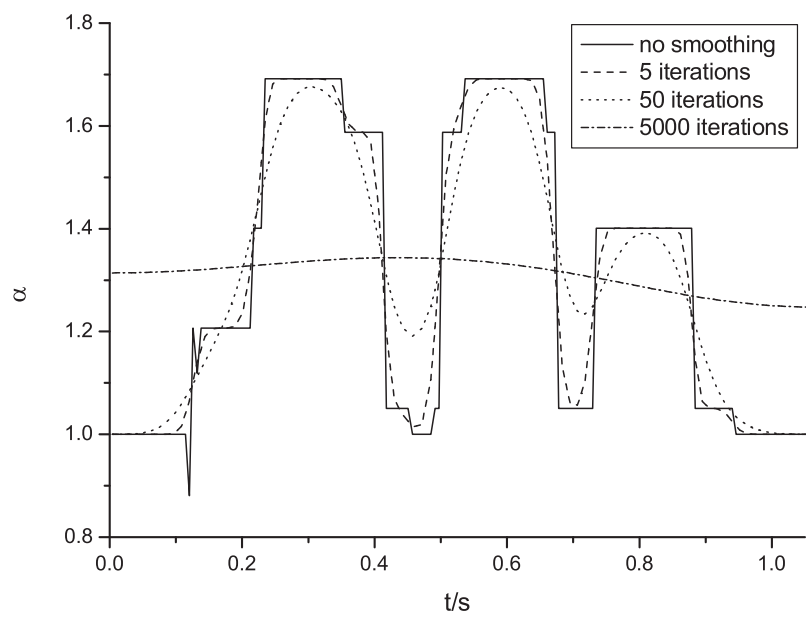

Fig. 2. Iterative Integrating Smoothing for Warping Functions with One Parameter.

After calculating the maximal distance between arbitrary complex spectra $X^{\prime}$ and $Y^{\prime}$ respectively real warping functions $\bar{\vartheta}^{\prime}$ and $\vartheta^{\prime}$, we obtain

$$
\begin{aligned}
d_{\vartheta}=\int_{\omega=0}^{\pi} & \left\{\frac{\lambda}{4 E_{0}}\left|Y(\omega)-X\left(\tilde{\omega}_{\vartheta}(\omega)\right)\right|^{2}\right. \\
& \left.+\frac{1-\lambda}{\pi^{3}}\left(\tilde{\omega}_{\bar{\vartheta}}(\omega)-\tilde{\omega}_{\vartheta}(\omega)\right)^{2}\right\} d \omega .
\end{aligned}
$$

\section{EXPERIMENTS}

Several experiments have been performed to investigate the properties of VTLN voice conversion with respect to the warping functions discussed in this paper. 
Three corpora of different languages and genders have been applied:

[A] 3 English sentences of a female speaker,

[B] 10 German sentences of a male speaker (poems),

[C] 3 German sentences of a male speaker (news).

In the following, we report results for three combinations of these corpora:

- F2M: female $[\mathrm{A}]$ is converted to male [B],

- M2F: male [B] is converted to female [A],

- M2M: male [C] is converted to male [B].

As error measure, we use the normalized class average distance

$$
d_{c a d}=\frac{\sum_{k=1}^{K_{S}} \int_{\omega=0}^{\pi}\left|\bar{Y}_{k}(\omega)-\bar{X}_{k}\left(\tilde{\omega}_{\vartheta_{k}}(\omega)\right)\right|^{2}}{4 K_{S} E_{0}} .
$$

Again, $\bar{X}$ and $\bar{Y}$ are spectra with unity energy $E_{0}$, consequently, we have $0 \leq d_{\text {cad }} \leq 1$ (cp. Section 5.2).

In Table 1, we show results for warping functions with one parameter (cf. Section 3). In the third row the results for the trivial solution $\tilde{\omega}=\omega$, i. e. no warping at all, is displayed to assess the absolute $d_{\text {cad }}$ values.

Table 1. Error Measure for Warping Functions with One Parameter

\begin{tabular}{|l|c|c|c|}
\hline \multirow{2}{*}{ warping function } & \multicolumn{3}{|c|}{ class average distance [\%] } \\
\cline { 2 - 4 } & F2M & M2F & M2M \\
\hline \hline no warping & 8.3 & 13.2 & 7.3 \\
\hline piece-wise linear & 6.0 & $\mathbf{6 . 4}$ & $\mathbf{6 . 2}$ \\
power & $\mathbf{5 . 2}$ & $\mathbf{6 . 4}$ & $\mathbf{6 . 2}$ \\
quadratic & 5.4 & 7.8 & $\mathbf{6 . 2}$ \\
bilinear & 5.5 & 6.5 & $\mathbf{6 . 2}$ \\
\hline
\end{tabular}

We note that the presented warping techniques do not essentially differ, but nevertheless, in our experiments, the power function consistently produced the best outcomes. The most significant effect was achieved for male-to-female voice conversion which is due to the large differences of the vocal tract. Concerning the above results, the opposite case is more complicated. This statement is also supported by our next experiments dealing with the piece-wise warping with several segments, vide Table 2

This table conspicuously demonstrates how the number of free parameters affects the warping precision. If $S$ becomes the number of spectral lines of the compared spectra, it passes into a variant of dynamic frequency warping with certain constraints. Nevertheless, subjective tests have shown, that excessively increasing the number of free parameters, results in an overfitting between source and target spectra and therewith disturbs the naturalness of the output speech.
Table 2. Error Measure for the Piece-Wise Warping Function with Several Segments

\begin{tabular}{|l|c|c|c|}
\hline \multirow{2}{*}{$S$} & \multicolumn{3}{|c|}{ class average distance [\%] } \\
\cline { 2 - 4 } & F2M & M2F & M2M \\
\hline \hline 1 & 6.7 & 7.6 & 6.3 \\
2 & 6.0 & 6.1 & 5.7 \\
4 & 5.4 & 5.0 & 5.1 \\
8 & 4.9 & 4.1 & 4.7 \\
16 & 4.5 & 3.4 & 4.0 \\
32 & 4.2 & 2.3 & 3.0 \\
64 & $\mathbf{4 . 1}$ & $\mathbf{1 . 4}$ & $\mathbf{2 . 3}$ \\
\hline
\end{tabular}

Future experiments are to investigate the consistency of the above results on other corpora. Furthermore, the overfitting effect is to be demonstrated using an adequate objective error criterion.

\section{REFERENCES}

[1] T. Kamm, G. Andreou, and J. Cohen, "Vocal tract normalization in speech recognition: Compensating for systematic speaker variability," in Proc. of the 15th Annual Speech Research Symposium, Baltimore, USA, 1995.

[2] E. Moulines and Y. Sagisaka, "Voice conversion: State of the art and perspectives," in Speech Communication, 16(2), 1995.

[3] O. Türk, "New methods for voice conversion," in $P h D$ Thesis, Boğaziçi University, Istanbul, Turkey, 2003.

[4] A. Kain and M. W. Macon, "Spectral voice transformations for text-to-speech synthesis," in Proc. of the ICASSP'98, Sydney, Australia, 1998.

[5] D. Sündermann and H. Ney, "An automatic segmentation and mapping approach for voice conversion parameter training," in Proc. of the AST'03, Maribor, Slovenia, 2003.

[6] L. F. Uebel and P. C. Woodland, "An investigation into vocal tract length normalization," in Proc. of the EUROSPEECH'99, Budapest, Hungary, 1999.

[7] E. Eide and H. Gish, "A parametric approach to vocal tract length normalization," in Proc. of the ICASSP'96, Atlanta, USA, 1996.

[8] M. Pitz, S. Molau, R. Schlüter, and H. Ney, "Vocal tract normalization equals linear transformation in cepstral space," in Proc. of the EUROSPEECH'01, Aalborg, Denmark, 2001.

[9] A. Acero and R. M. Stern, "Robust speech recognition by normalization of the acoustic space," in Proc. of the ICASSP'91, Toronto, Canada, 1991. 